\title{
A Case of Amiodarone Pulmonary Toxicity with Short-term Amiodarone Use
}

\author{
Abigail McDonald ${ }^{1}$ \\ 1. Internal Medicine, HCA Healthcare/USF Morsani College of Medicine GME Programs, Northside Hospital, St. \\ Petersburg, USA
}

Corresponding author: Abigail McDonald, abigailestella@gmail.com

\begin{abstract}
This is the case of a 92-year-old female who was hospitalized one month prior to admission for symptomatic paroxysmal atrial fibrillation, requiring intravenous amiodarone. Following her previous admission, she was placed on one month of amiodarone $200 \mathrm{mg}$ twice daily, with a one week transition to $200 \mathrm{mg}$ daily. The patient subsequently developed progressive shortness of breath and dry cough over a period of several weeks. Initial imaging showed diffuse bilateral coarse patchy interstitial infiltration on chest X-ray and bibasilar pleural effusions and scattered bilateral opacities on CTA chest. In an elderly patient presenting with dyspnea and dry cough, as well as interstitial opacities on imaging, amiodarone pulmonary toxicity should be considered despite short-term low-dose use.
\end{abstract}

Categories: Internal Medicine, Infectious Disease

Keywords: amiodarone pulmonary toxicity

\section{Introduction}

Amiodarone is a frequently used drug for the management of arrhythmia. It is associated with various pulmonary adverse effects, including but not limited to interstitial fibrosis, hypersensitivity pneumonitis, acute respiratory distress syndrome, and alveolitis. Clinical presentations include gradually worsening dyspnea, dry cough, and pleuritic chest pain [1]. They may also include fevers, weight loss, and hemoptysis [2]. Adverse pulmonary effects may be observed as rapidly as following the first few days of amiodarone use to chronically and insidiously over years [3]. One recent case report of an elderly woman prescribed $400 \mathrm{mg}$ three times daily over two weeks and $200 \mathrm{mg}$ daily thereafter was readmitted within three weeks with symptoms of toxicity [4]. Another recent case report of a 68-year-old female status-post triple vessel coronary artery bypass grafting complicated by atrial fibrillation who received bolus intravenous amiodarone and was discharged on $400 \mathrm{mg}$ PO amiodarone returned to the hospital after 10 days with symptoms of toxicity [5]. Adverse effects may be seen at doses as low as $200 \mathrm{mg}$ daily [6]. Risk factors are not well-defined but may include increasing age, underlying pulmonary disease, cumulative doses, and doses greater than $400 \mathrm{mg}$ [2].

Received 03/14/2020

Review began 04/02/2020 Review ended 04/10/2020 Published 04/15/2020

(c) Copyright 2020 McDonald. This is an open access article distributed under the terms of the Creative Commons Attribution License CC-BY 4.0., which permits unrestricted use, distribution, and reproduction in any medium, provided the original author and source are credited.

\section{Case Presentation}

A 92-year-old non-smoker female with a past medical history of paroxysmal atrial fibrillation on apixaban and metoprolol, tachycardia-bradycardia syndrome with a St. Jude dual-chamber permanent pacemaker, insomnia, hyperlipidemia, hypertension, gastroesophageal reflux disease, and osteoarthritis presented for the evaluation of progressively worsening shortness of breath for the last two to three weeks with acute worsening on the night of admission after being out dancing. The patient had just recently been hospitalized one month and a week prior for symptomatic paroxysmal atrial fibrillation, requiring intravenous (IV) amiodarone and discharged on a regimen of amiodarone $200 \mathrm{mg}$ twice daily for one month, then $200 \mathrm{mg}$ once daily for the last week. The patient reported that even after taking a shower, she feels winded. She additionally reported progressively worsening dry cough and wheezing over the last two to three weeks. The patient denied hemoptysis, productive cough, palpitations, chest pain, orthopnea, lower extremity edema, fevers, chills, weakness, dizziness, and recent illness. The patient additionally reported that since starting amiodarone, she developed blurred vision and tremors that were progressively worsening. The patient reported undergoing recent outpatient pulmonary function testing, which was unremarkable.

In the emergency department, the patient's initial oxygen saturation was $85 \%$ and she was mildly tachypneic. The patient was started on a non-rebreather mask, then weaned to a $2 \mathrm{~L}$ nasal cannula with improvement to oxygen saturation of $96 \%$. The initial chest X-ray showed diffuse bilateral coarse patchy interstitial infiltrates and pleural effusions (Figure 1). 


\section{Cureus}

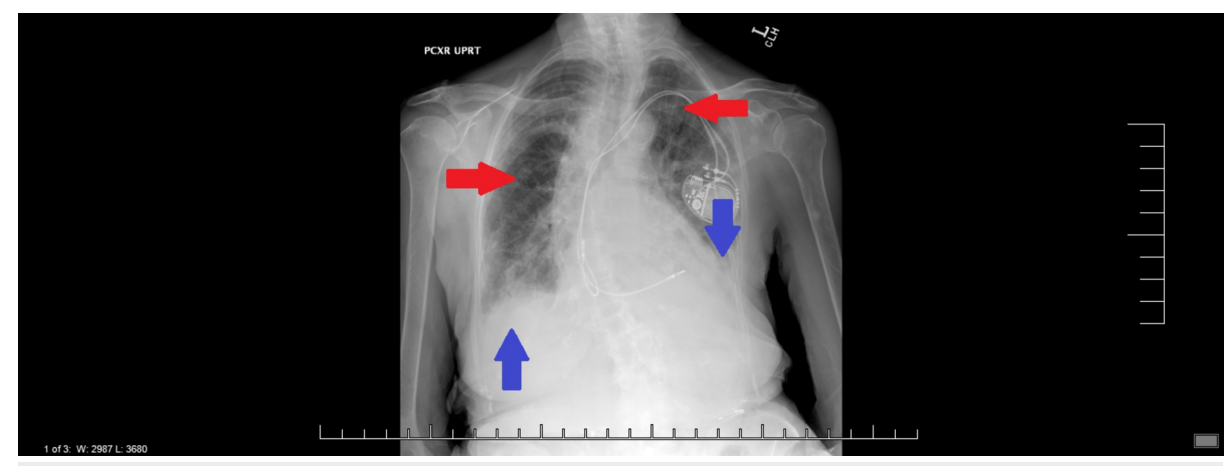

\section{FIGURE 1: Chest X-ray}

Diffuse bilateral coarse patchy interstitial infiltrates and pleural effusions

Red arrows - interstitial infiltrate, blue arrows - pleural effusions

In the emergency department, D-dimer was found to be elevated and the ensuing bilateral lower extremity venous duplex scan was negative for lower extremity deep vein thrombosis. Computerized tomography angiography of the chest showed no evidence for pulmonary embolism, though bibasilar pleural effusions were noted in addition to scattered bilateral opacities with possible early signs of honeycombing (Figure 2).

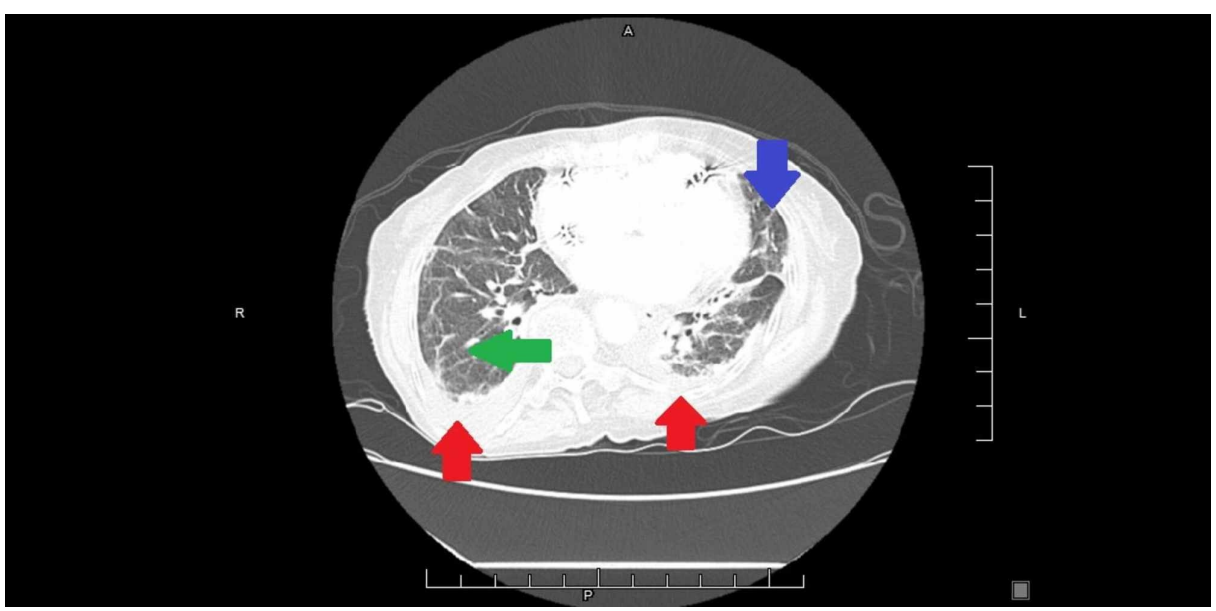

FIGURE 2: CT angiography of the chest

Red arrow - pleural effusion, green arrow - opacity, blue arrow - honeycombing

Upon admission, the physical exam was remarkable for bilateral crackles with a Velcro-like quality from the bases to the mid-lung. No wheezing was appreciated. The cardiac exam was unremarkable - the patient had regular rate and rhythm, no murmurs were auscultated, no pitting edema, jugular vein distention (JVD), or carotid bruits noted. The patient was afebrile and appeared to be in no acute distress. The thyroid was not enlarged with no palpable irregularities. The ophthalmic exam was unremarkable. The skin color was noted to be normal. Initial labs showed no leukocytosis and arterial blood gas was consistent with a primary respiratory alkalosis. Troponin was negative, NT-pro-B-type natriuretic peptide was elevated, and procalcitonin was negative. The electrocardiogram (ECG) demonstrated no acute ischemic changes or arrhythmias - heart rate was within normal limits, PR and QT intervals were not elongated, and QRS was narrow (Figure 3). 


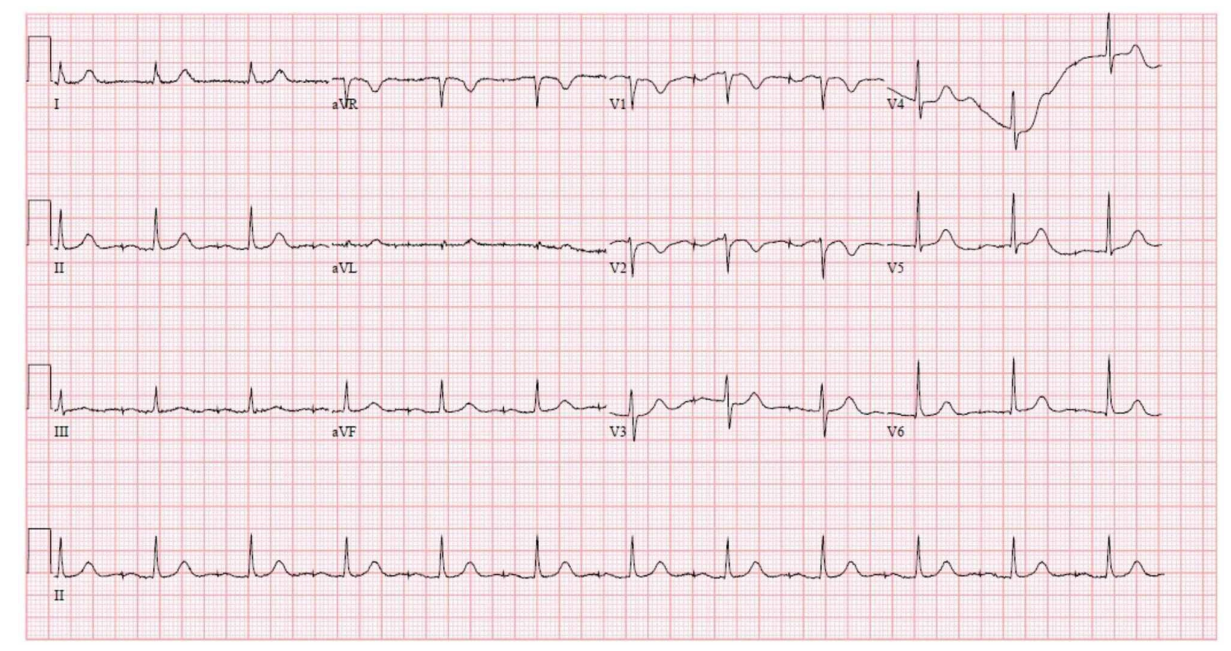

FIGURE 3: ECG - atrial-paced rhythm, no acute ischemic changes

ECG: electrocardiogram

The echocardiogram showed normal systolic function and the ejection fraction was $60 \%-65 \%$.

The patient's amiodarone was stopped and she was given supportive treatment and started on prednisone given concern for possible amiodarone toxicity. As atypical pneumonia could not be wholly ruled out, the patient was started on levofloxacin. The patient subsequently developed urticaria and was switched to doxycycline. Legionella antigen was negative, Mycoplasma pneumonia immunoglobulin M (IgM) was nonreactive, and urine Streptococcus pneumoniae antigen was negative.

The six-minute walk test revealed $87 \%$ oxygen saturation on room air at rest and $85 \%$ with activity, with improvement to $94 \%$ at rest and with activity on a $2 \mathrm{~L}$ nasal cannula. The patient reported ongoing dyspnea on exertion, though improvement was found as the patient was weaned to a $1 \mathrm{~L}$ nasal cannula with good saturation. A follow-up chest X-ray prior to discharge continued to show coarse interstitial opacities.

\section{Discussion}

In an early, single-center study of 80 patients being treated with amiodarone for refractory arrhythmias, $5 \%$ developed the clinical and radiologic features of interstitial pneumonitis, having no prior history of lung disease. Of these patients with interstitial pneumonitis, $75 \%$ were receiving a maintenance dose of $400 \mathrm{mg}$ daily over five to 36 months [1]. One study of 573 patients in a single-center study found the incidence of amiodarone pulmonary toxicity to be $5.8 \%$, occurring between six days and 60 months of treatment, the highest incidence occurring within the first 12 months. Patients older than 62 years developed toxicity more frequently. Patients who started therapy younger than the age of 40 did not develop toxicity in this study. Other risk factors included lower pre-amiodarone treatment diffusing capacity for carbon monoxide (DLCO) and higher daily average maintenance dose. The development of toxicity was not predicted with factors, including gender, arrhythmia, lung volume abnormalities, or underlying heart disease [7]. In this case, the patient was at risk given her age and incidence of possible amiodarone toxicity occurring within her first 12 months of therapy.

Several possible mechanisms are proposed regarding amiodarone pulmonary toxicity. One such mechanism is via inflammatory or immune effector cells. Another mechanism is direct toxicity resulting in lung parenchymal cell injury [8]. One study indicated that in vitro amiodarone could induce bovine pulmonary artery endothelial cells to form lamellar cytoplasmic inclusions, occurring as soon as four hours after incubation with amiodarone with as little as 1 microgram/ml [9]. Another study found pulmonary fibrosis to be mediated by the dose-dependent induction of apoptosis and necrosis in vitro (rat alveolar epithelial cells and human-derived alveolar epithelial cells). Cytotoxicity was significant at below-therapeutic concentrations of amiodarone and severe at concentrates known to accumulate in human lung tissue. Interestingly, apoptosis was inhibited by captopril (angiotensin-converting enzyme (ACE) inhibitor) and saralasin (partial angiotensin II receptor agonist) [10].

At present, there is no gold standard for the diagnosis of amiodarone-induced pulmonary toxicity. Other causes must be ruled out, including other pulmonary conditions such as chronic obstructive pulmonary disease (COPD) or pulmonary infarct, pulmonary embolism, infectious, cardiac, and malignant (carcinoma, lymphoma). Pulmonary function testing demonstrates restrictive defects with reduced diffusion capacity [1]. One study found a median TLC of $78 \%$, with diffusion defects in $45 \%$ of participants. TLC and DLCO can 
improve but not significantly in the long term. VC, however, can improve significantly between one and 36 months. A small number of patients may have persistent restrictive syndromes. A chest X-ray can show bilateral opacifications. A resolution of findings can occur over two to eight weeks. High-resolution CT can reveal diffuse bilateral ground-glass opacification, alveolar consolidation, intra-alveolar reticulations, and pleural effusions [11]. In this case, patient history, exam, and workup, including ECG, troponin, and echocardiogram, make cardiac pathology less likely. Recent outpatient pulmonary function testing was negative, though it may be of consideration to obtain new testing given that the patient has significant imaging findings and clinical complaints. Extensive infectious workup is being pursued and prophylactic antibiotics are prudent as atypical pneumonia cannot be completely ruled out at this time. Clinical presentation, in addition to imaging findings, is less likely to support malignancy.

At present, there are no controlled trials to demonstrate the efficacy of any given treatment. Amiodarone cessation can result in the improvement of clinical presentation over two to eight weeks [1]. Imaging and pulmonary function do not tend to improve as quickly as clinical presentation. Discontinuation alone may be sufficient if the disease is limited. In patients who show a substantial involvement on imaging studies, or if patients are hypoxic, systemic corticosteroids may be utilized [12]. Treatment with systemic corticosteroids was found in one study to induce relapse when treatment lasted for less than three months but not longer than six months, probably attributable to the 40-70 day half-life elimination of amiodarone and storage in the lung parenchyma. Positive effects were noted on alveolar opacifications [11]. An initial dose of 0.75-1 $\mathrm{mg} / \mathrm{kg}$ of prednisolone should be maintained until there is a definite clinical and radiographic response, as recurrence can be more severe than the initial episode. If no improvement is found after one to two months of systemic corticosteroids, alternative diagnoses should be considered. Unfortunately, amiodarone-induced fibrosis can be irreversible with poor response to corticosteroids. Acute respiratory distress syndrome (ARDS) in the setting of amiodarone toxicity is rapidly progressive, often requiring mechanical ventilation, and is also found to have a poor response to systemic corticosteroid therapy [12]. Another study investigating the effects of captopril and the angiotensin receptor antagonist losartan found that alveolar wall collagen accumulation was significantly reduced by the administration of captopril (100\%) or losartan (70\%), but these agents had no effect on the accumulation of alveolar macrophages stimulated by amiodarone, suggesting an effect on fibrosis and not alveolitis [13]. A following eight-year retrospective single-center analysis of 1000 patients on amiodarone was conducted, finding amiodarone pulmonary toxicity in $2.2 \%$ of that population, with the occurrence of toxicity in $1 \%$ of patients already on an ACE-I or angiotensin II receptor blocker (ARB) and in 3.9\% of patients not taking an ACE-I or an ARB, suggesting a possible protective effect of ACE-Is/ARBs against amiodarone pulmonary toxicity [14]. A case study conducted on a patient with severe toxicity and rapid onset respiratory failure refractory to corticosteroid therapy on mechanical ventilation was started on polymyxin B-immobilized fiber column direct hemoperfusion (PMX-DHP), a device that reduces blood endotoxin levels generally used in cases of sepsis. Clinical improvement of respiratory failure was observed with a reduction in the serum levels of amiodarone and its metabolite. The patient did well without recurrence [15]. If no infectious source is found, this patient may benefit from continued systemic corticosteroid treatment for another one to two months with reevaluation for clinical and imaging improvement. As the patient has failed her six-minute walk test, she will need to go home with oxygen therapy. Given the patient's history of hypertension, the initiation of an ACE inhibitor or ARB may be considered for protection against worsening fibrosis. The patient will need to remain off of amiodarone therapy, and alternative medications to protect against arrhythmia may be pursued.

\section{Conclusions}

Although the incidence of amiodarone pulmonary toxicity is relatively low, especially with dosing less than $400 \mathrm{mg}$ daily, its possibility in an elderly patient should be considered. Adverse effects have been noted with amiodarone at doses as low as $200 \mathrm{mg}$ daily and as soon as within the first few days of amiodarone use. Additionally, cytotoxicity has been noted at below-therapeutic concentrations. Future studies should be pursued to determine the efficacy of treatments for amiodarone pulmonary toxicity, standardizing diagnosis, as well as better characterizing amiodarone pulmonary toxicity in the setting of short-term therapy.

\section{Additional Information \\ Disclosures}

Human subjects: Consent was obtained by all participants in this study. Conflicts of interest: In compliance with the ICMJE uniform disclosure form, all authors declare the following: Payment/services info: All authors have declared that no financial support was received from any organization for the submitted work. Financial relationships: McDonald A declare(s) NA from HCA Healthcare and/or an HCA Healthcare-affiliated entity. This research was supported (in whole or in part) by HCA Healthcare and/or an HCA Healthcare affiliated entity. The views expressed in this publication represent those of the author(s) and do not necessarily represent the official views of HCA Healthcare or any of its affiliated entities. Other relationships: All authors have declared that there are no other relationships or activities that could appear to have influenced the submitted work. 


\section{References}

1. McGovern B, Garan H, Kelly E, Ruskin JN: Adverse reactions during treatment with amiodarone hydrochloride. Br Med J. 1983, 287:175-180. 10.1136/bmj.287.6386.175

2. Merino JL, Perez de Isla P L: Treatment with amiodarone: how to avoid complications . E-Journal of the European Society of Cardiology Council for Cardiology Practice. 2011, 10:[Epub].

3. Papiris SA, Triantafillidou C, Kolilekas L, Markoulaki D, Manali ED: Amiodarone: review of pulmonary effects and toxicity. Drug Saf. 2010, 33:539-558. 10.2165/11532320-000000000-00000

4. Tanayan C, Janda T, Ilkanich C, Pastoral M, Silver K: Amiodarone toxicity: sooner than you expect. J Am Coll Cardiol. 2019, 73:2938. 10.1016/S0735-1097(19)33544-2

5. Uyanga B, Rajasekaran A, Mellon J: Short course of amiodarone-induced severe lung toxicity in post-CABG patient. Am J Respir Crit Care Med. 2017, 195:5532.

6. Ott MC, Khoor A, Leventhal JP, Paterick TE, Burger CD: Pulmonary toxicity in patients receiving low-dose amiodarone. Chest. 2003, 123:646-651. 10.1378/chest.123.2.646

7. Dusman RE, Stanton MS, Miles WM, Klein LS, Zipes DP, Fineberg NS, Heger JJ: Clinical features of amiodarone-induced pulmonary toxicity. Circulation. 1990, 82:51-59. 10.1161/01.CIR.82.1.51

8. Martin WJ: Mechanisms of amiodarone pulmonary toxicity. Clin Chest Med. 1990, 11:131-138.

9. Martin WJ, Howard DM: Amiodarone-induced lung toxicity. In vitro evidence for the direct toxicity of the drug. Am J Pathol. 1985, 120:344-350.

10. Bargout R, Jankov A, Dincer E, et al.: Amiodarone induces apoptosis of human and rat alveolar epithelial cells in vitro. Am J Physiol Lung Cell Mol Physiol. 2000, 278:1039-1044. 10.1152/ajplung.2000.278.5.L1039

11. Mankikian J, Favelle O, Guillon A, et al.: Initial characteristics and outcome of hospitalized patients with amiodarone pulmonary toxicity. Respir Med. 2014, 108:638-646. 10.1016/j.rmed.2014.01.014

12. Schwaiblmair M, Berghaus T, Haeckel T, Wagner T, von Scheidt W: Amiodarone-induced pulmonary toxicity: an under-recognized and severe adverse effect?. Clin Res Cardiol. 2010, 99:693-670.

13. Uhal BD, Wang R, Laukka J, Zhuang J, Soledad-Conrad V, Filippatos G: Inhibition of amiodarone-induced lung fibrosis but not alveolitis by angiotensin system antagonists. Pharmacol Toxicol. 2003, 92:81-87. 10.1034/j.1600-0773.2003.920204.x

14. Kosseifi SG, Halawa A, Bailey B, Micklewright M, Roy TM, Byrd RP Jr: Reduction of amiodarone pulmonary toxicity in patients treated with angiotensin-converting enzyme inhibitors and angiotensin receptor blockers. Ther Adv Respir Dis. 2009, 3:289-294. 10.1177\%2F1753465809348015

15. Sato N, Kojima K, Horio Y, Goto E, Masunaga A, Ichiyasu H, Kohrogi H: Successful treatment of severe amiodarone pulmonary toxicity with polymyxin B-immobilized fiber column direct hemoperfusion. Chest. 2013, 143:1146-1150. 10.1378/chest.12-0994 